\title{
¿Hay diferencia en la calidad de vida en adultos con parálisis cerebral gross motor function classification system (GMFCS) IV-V, tratados con reconstrucción de cadera vs osteotomía varodesrotadora sin cotiloplastia durante la infancia?
}

\author{
Is there any life quality difference in adults with cerebral paralysis, gross motor \\ function classification system (GMFCS) IV-V, treated with hip reconstruction \\ vs varus derotation osteotomy without cotiloplasty during childhood? \\ Dr. Ignacio Osvaldo Fortis Olmedo, ${ }^{*}$ Dr. Javier José Ardón Dubón, ${ }^{\star}$ Dr. Jorge Gómez Chavarría, ${ }^{\ddagger}$ \\ Dr. Luis Nualart Hernández, ${ }^{\ddagger}$ Dra. Gabriela Ávalos Arroyo ${ }^{\S}$ \\ Hospital Shriners para Niños México. \\ * Médico residente del Hospital Shriners para Niños, México. \\ ¥ Médico Staff del Hospital Shriners para Niños, México. \\ ${ }^{\S}$ Médico residente en Hospital General Regional número 1 del Instituto Mexicano del Seguro Social, Querétaro. México.
}

\section{RESUMEN}

La parálisis cerebral (PC) es un grupo de alteraciones permanentes del desarrollo del movimiento y postura que causan limitación psicomotriz. El desplazamiento de la cadera es la segunda deformidad musculoesquelética más común después del equino. El tratamiento dependerá del grado del GMFCS y de los hallazgos clínicos y radiográficos. Objetivo: Analizar la calidad de vida en pacientes adultos con PC GMFCS IV-V que se trataron con reconstrucción de cadera contra los que fueron tratados únicamente con tejidos blandos y osteotomía varodesrotadora (OVD) durante la infancia antes de los 18 años de edad. Estudio descriptivo, retrospectivo, transversal y observacional de los pacientes con diagnóstico de PC variedad espástica gross motor function classification system IV-V, con una edad mínima actual de 27 años. La calidad de vida fue evaluada con cuatro escalas, SF-36 versión 2, QoLIBRI, CAVIDACE y GENCAT sin diferencia significativa entre ambos grupos.

\section{ABSTRACT}

Cerebral palsy $(C P)$ is a group of permanent alterations in the development of movement and posture, which cause psychomotor limitation. Hip displacement is the second most common musculoskeletal deformity after the equine. Treatment will depend on the degree of GMFCS and clinical and radiographic findings. The aim of this trail was analyze the quality of life in adult patients with $C P$ GMFCS IV-V, who were treated with hip reconstruction against those who were only treated with soft tissues and VDO during childhood before 18 years of age. Descriptive, retrospective, cross-sectional and observational study of patients with a diagnosis of CP spastic variety gross motor function classification system IV-V, with a current minimum age of 27 years. Quality of life was evaluated with 4 scales, SF-36 v2, QoLIBRI, CAVIDACE and GENCAT without significant difference between both groups. 
Palabras clave: Parálisis cerebral, calidad de vida, osteotomía varodesrotadora, cotiloplastia, adulto, QoL, cadera espástica.

Nivel de evidencia: IV
Keywords: Cerebral palsy, life quality, varus derotation osteotomy, cotiloplasty, adult, QoL, spastic hip.

Evidence level: IV

\section{INTRODUCCIÓN}

La parálisis cerebral (PC) es un grupo de alteraciones permanentes del desarrollo del movimiento y postura que causan limitación psicomotriz. Se atribuye a una serie de alteraciones no progresivas que ocurren en el desarrollo del cerebro fetal o infantil de tipo isquémico o hemorrágico. ${ }^{1}$ Los desórdenes motores se acompañan de alteraciones en la sensación, percepción, cognición, comunicación, conducta y problemas musculoesqueléticos. ${ }^{1}$ La lesión del cerebro en desarrollo puede ocurrir en cualquier momento desde la gestación hasta la primera infancia y se clasifica como prenatal, perinatal o postnatal, siendo el periodo prenatal el más frecuente. ${ }^{2}$ La PC es la causa más común de discapacidad física infantil en los países desarrollados con una incidencia de dos por 1,000 nacidos vivos. ${ }^{3}$ Se caracteriza por una lesión que ocurre en el cerebro inmaduro que desarrolla un trastorno permanente del tono muscular y del control motor, generalmente es progresiva, sus características principales son contracturas musculares extensas, deformidad torsional ósea e inestabilidad de las articulaciones. El desplazamiento de la cadera es la segunda deformidad musculoesquelética más común después del equino, el cual afecta a más de un tercio de los niños con parálisis cerebral. ${ }^{3}$

La cadera luxada o subluxada en los pacientes con diagnóstico de PC resulta un problema serio para los afectados y/o sus cuidadores. ${ }^{4}$ La diferencia en el tratamiento de la cadera espástica en pacientes con PC es inmensa y suele individualizarse. ${ }^{5}$ La historia natural de la cadera en los pacientes con PC dependerá del grado de afección motor gruesa (gross motor function classification system, GMFCS por sus siglas en inglés) en la que se encuentre el paciente, de esta forma, en pacientes que tienen un GMFCS 1 el porcentaje de desplazamiento de la cadera será menor de $6 \%$, mientras que en pacientes con GMFCS IV-V el riesgo de subluxación o luxación será de $69-80 \%$ de los casos. ${ }^{6}$ El desarrollo de la deformidad de cadera en pacientes espásticos es el resultado de las fuerzas musculares ejercidas a dicha articulación, manifestándose como anteversión, coxa valga, displasia acetabular, oblicuidad pélvica así como subluxación o luxación de la cadera. ${ }^{4}$ La presencia del dolor en edades tempranas es la principal causa de tratamiento en pacientes no deambuladores (GMFCS IV-V). ${ }^{7}$

El tratamiento dependerá del grado del GMFCS y de los hallazgos clínicos y radiográficos (subluxación de la cadera «índice de Reimers») con los que cuente el paciente. ${ }^{6}$ Robb y colaboradores recomiendan realizar una vigilancia con rayos $X$ cada seis meses hasta los siete años y posteriormente anual hasta la madurez esquelética. ${ }^{6}$ Grosso modo la cadera espástica suele manejarse sólo con observación si el índice de Reimers es menor de $30 \%$ y quirúrgico si es mayor de $30 \% .^{8}$ El manejo quirúrgico dependerá del grado de subluxación/luxación con el que cuente el paciente:

1. Tratamiento profiláctico alargamiento de tejidos blandos (miotomía de aductor mediano y tenotomía del psoas [MAP]): pacientes con cadera en riesgo con un Reimers $>30 \%$, abducción $<30-45^{\circ}$, incremento mayor de $10 \%$ de migración en menos de un año. ${ }^{9}$

2. Cirugía de reconstrucción (alargamiento de tejidos blandos más osteotomía varodesrotadora [OVD] con o sin acortamiento femoral, más acetabuloplastia): pacientes menores de ocho años con Reimers > $50 \%$ o con cirugía profiláctica fallida (porcentaje de migración mayor de 40\% en un año de postoperado); pacientes mayores de ocho años con Reimers mayor de $40 \%$ con ausencia de cambios degenerativos avanzados. ${ }^{9}$

3. Cirugía de salvamento: caderas luxadas dolorosas con cambios degenerativos avanzados. Entre las opciones se encuentran la osteotomía de Castle, las osteotomías de redirección (osteotomías valguizantes), artroplastia de interposición (McHale), la artrodesis o el reemplazo articular. ${ }^{10}$

Existen estudios ${ }^{11}$ que demuestran que la calidad de vida de los pacientes adultos con PC es mala en comparación con la población en general. El objetivo de este estudio es analizar la calidad de vida en pacientes adultos con PC GMFCS IV-V sometidos a reconstrucción de cadera (alargamiento de tejidos blandos más osteotomía varodesrotadora [OVD] con o sin acortamiento femoral, más acetabuloplastia) contra los que fueron tratados únicamente con alargamiento de tejidos blandos y OVD durante la infancia antes de los 18 años de edad.

\section{MATERIAL Y MÉTODOS}

Se realizó un estudio descriptivo, retrospectivo, transversal y observacional de los pacientes que cumplieron con los siguientes criterios de inclusión: todos aquellos pacientes con diagnóstico de parálisis cerebral variedad espástica GMFCS 
IV-V, dados de alta por edad en el Hospital Shriners para Niños de la Ciudad de México con una edad mínima actual de 27 años al momento del estudio, y en los que se haya practicado una cirugía de reconstrucción de cadera (alargamiento de tejidos blandos más OVD con o sin acortamiento femoral, más acetabuloplastia) o se les haya tratado sólo con OVD y alargamiento de tejidos blandos sin acetabuloplastia antes de los 18 años de edad. Se excluyeron todos los pacientes que no cumplieron los criterios de inclusión, todos los pacientes con más de una cirugía de cadera, pacientes con parálisis diferentes a la espástica, pacientes con expediente incompleto, o pacientes a los que se les sometió a cirugía de salvamento. Los datos fueron obtenidos de los expedientes clínicos de la clínica de parálisis cerebral de nuestro hospital y de un interrogatorio vía telefónica con los principales cuidadores de los pacientes.

Se aplicaron los siguientes cuestionarios a los cuidadores principales de cada paciente: cuestionario de salud SF-36 versión 2, cuestionario QoLIBRI (Quality of
LIfe after BRain Injury [calidad de vida posterior a la lesión cerebral]), CAVIDACE (Evaluación de la CAlidad de VIda de personas con DAño CErebral), y GENCAT quality of life scale (Generalitat de Catalunya [Gobierno de Cataluña]). El análisis estadístico se realizó con el programa SPSS 23, se determinó un intervalo de confianza de 95\%, y se calculó un valor significativo $p<0.05$

\section{RESULTADOS}

Se obtuvieron 2,115 pacientes (Figura 1) con PC, los cuales fueron dados de alta del Hospital Shriners de México en el periodo comprendido de 1998 a 2008, con una edad mínima actual de 27 años, de los cuales 1889 eran de la variedad espástica. Sólo 356 pacientes fueron clasificados como GMFCS IV-V y de ellos 178 presentaban afecciones en caderas, 56 pacientes fueron tratados sólo con alargamiento de tejidos blandos + OVD y de los cuales 27 tenían expediente clínico y radiográfico completo (grupo 1). De los

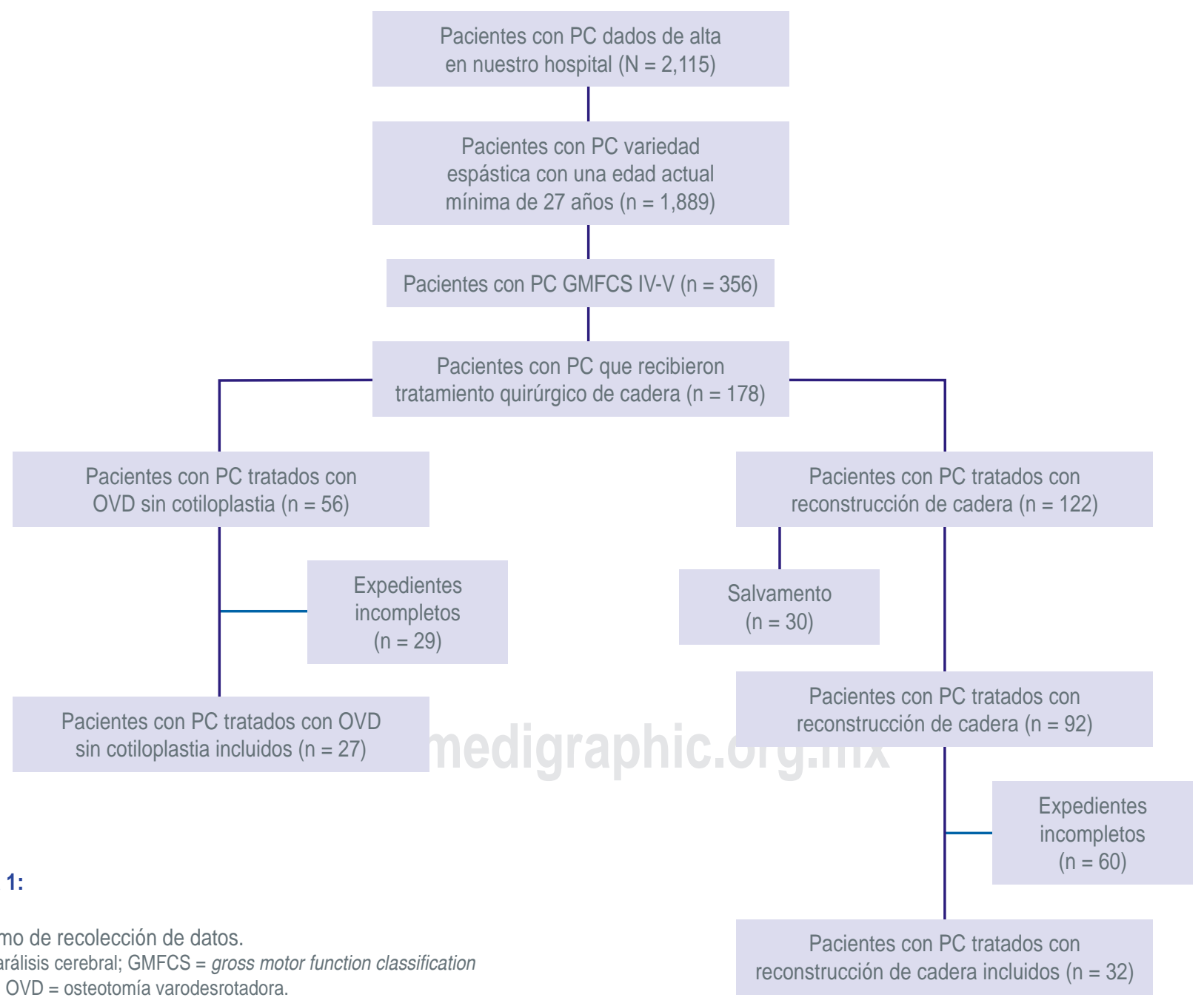


Tabla 1: Comparación entre ambos grupos sometidos a los cuestionarios de calidad de vida.

\begin{tabular}{lccc}
\hline Escala aplicada & Grupo 1 & Grupo 2 & $p$ \\
SF-36 versión 2 & $15.6(7.3-22.5)$ & $16.2(8.3-19.8)$ & 0.45 \\
QoLIBRI & $6.6(5.6-9.3)$ & $5.9(4.5-7.2)$ & 0.35 \\
CAVIDACE (percentila) & $5(1-15)$ & $5(1-10)$ & 0.67 \\
CAVIDACE (índice de calidad de vida) & $66(10-85)$ & $73(12-81)$ & 0.09 \\
GENCAT (percentila) & $5(1-10)$ & $5(1-10)$ & 0.45 \\
GENCAT (índice de satisfacción) & $57(12-83)$ & $53(17-77)$ & 0.38 \\
\hline
\end{tabular}

Prueba $U$ de Mann-Whitney.

pacientes, 122 recibieron tratamiento quirúrgico (92 con reconstrucción de cadera y 30 con cirugía de salvamento), de los cuales 32 (grupo 2) contaban con expediente clínico y radiográfico completo tratados con reconstrucción de cadera (alargamiento de tejidos blandos más OVD con o sin acortamiento femoral, más cotiloplastia). Los pacientes con tratamiento de ambas caderas fueron eliminados del estudio. En el grupo 1 se incluyeron 27 pacientes, 13 fueron hombres y 14 mujeres, se estudiaron 25 pacientes con cuadriplejía y dos con paraplejía. La edad al momento de la cirugía: $<$ cinco años $=$ dos, de cinco a ocho años $=$ 11, de nueve a 12 años = ocho, de 13-17 años= seis. La edad promedio de la población al momento del estudio fue 30.2 años, 14 pacientes se clasificaron como GMFCS IV y 13 como GFMCS V. Se clasificó a los pacientes con el grado de espasticidad de Ashworth, siete pacientes se clasificaron como grado III, 14 como grado IV y seis como grado $\mathrm{V}$. En el grupo 2 se incluyeron 32 pacientes, 14 hombres y 18 mujeres, se estudiaron 27 pacientes con cuadriplejía, cuatro con paraplejía y una con hemiplejía (derecha). La edad al momento de la cirugía: < cinco años = cuatro, de cinco a ocho años $=14$, de nueve a 12 años $=$ nueve, de 13 a 17 años = cinco. La edad promedio de la población al momento del estudio fue 31.5 años, 13 pacientes se clasificaron como GMFCS IV y 19 como GFMCS V. Se clasificó a los pacientes con el grado de espasticidad de Ashworth, nueve pacientes se clasificaron como grado III, 15 como grado IV y ocho como grado V.

La media en el puntaje obtenido en el cuestionario SF-36 versión 2 para el grupo 1 fue de 15.6 puntos, mientras que para el grupo 2 fue de 16.2 puntos. El puntaje obtenido para el cuestionario QoLIBRI en el grupo 1 fue de una media de 6.6 puntos, mientras que para el grupo 2 fue de 5.9 puntos. Por otra parte, la percentila obtenida en el cuestionario CAVIDACE para ambos grupos fue de 5. El índice de calidad de vida medido por el cuestionario CAVIDACE fue de 66 puntos para el grupo 1 y de 73 puntos para el grupo 2. La percentila obtenida en el cuestionario GENCAT para ambos grupos fue de 5. El índice de calidad de vida medido por el cuestionario GENCAT fue de 57 puntos para el grupo 1 y de 53 puntos para el grupo 2 (Tabla 1).

\section{DISCUSIÓN}

El principal objetivo de este estudio fue analizar la calidad de vida en pacientes adultos con PC GMFCS IV-V sometidos a reconstrucción de cadera contra los que fueron tratados sólo con alargamiento de tejidos blandos y OVD durante la infancia antes de los 18 años de edad. El tratamiento de la cadera espástica en el paciente pediátrico es en la actualidad un tema de continuo debate, ya que por la propia historia natural de la enfermedad, esta articulación tiende a luxarse y a ser dolorosa principalmente en pacientes con un GMFCS por arriba del grado III. EI principal objetivo de operar una cadera en el paciente espástico es evitar la luxación y subsecuentemente el dolor que se manifiesta en las caderas luxadas. ${ }^{12}$ También se ha comprobado que muchas de las caderas que se tratan con OVD más cotiloplastia o sin cotiloplastia presentan reluxación recidivante a pesar de una buena técnica quirúrgica; ${ }^{12}$ sin embargo, actualmente no hay un estudio que muestre la calidad de vida de estos pacientes en la etapa adulta.

En nuestro estudio la calidad de vida fue evaluada con cuatro escalas, SF-36 versión 2, QoLIBRI, CAVIDACE y GENCAT. El SF-36 fue construido para representar los conceptos de salud más importantes divididos en sus apartados, este cuestionario puede aplicarse al paciente o al familiar más cercano. Los resultados se expresan desde cero puntos (peor calidad de vida) hasta 100 puntos (mejor calidad de vida). En este cuestionario encontramos que ambos grupos muestran una calidad de vida mala, lo que favorece parcialmente al grupo 2, pero sin diferencia significativa entre ambos $(p=0.45)$. El cuestionario QoLIBRI fue diseñado para evaluar la calidad de vida en pacientes que han sufrido un daño cerebral de tipo isquémico o hemorrágico, cuenta con seis apartados que evalúan función, independencia y dolor. Los resultados en esta escala se representan desde cero $=$ peor calidad de vida hasta 100, la mejor calidad de vida posible. En este cuestionario se favorece parcialmente al grupo 1 ; sin embargo, no hay una diferencia significativa $(p=0.35)$. El cuestionario CAVIDACE se basa en el modelo de ocho dimensiones de Schalock y Verdugo de 2002, se aplica a pacientes mayo- 
res de 18 años con daño cerebral adquirido o congénito y validado por el Instituto Universitario de Integración en la Comunidad (INICO), Universidad de Salamanca. Este cuestionario incluye 64 ítems, los resultados se expresan en índice de calidad de vida que va desde $<68$ puntos (calidad de vida mala) hasta más de 131 puntos (excelente calidad de vida); y en percentiles: los percentiles indican el porcentaje de personas que tienen una puntuación superior o inferior. Cuanto mayor es el percentil obtenido, mayor es el nivel de calidad de vida. En nuestro estudio encontramos que no existe una diferencia significativa entre ambos grupos con este cuestionario (índice de calidad de vida $p=0.67$ y percentiles 0.09). De igual forma, la escala GENCAT desarrollada en Cataluña evalúa la calidad de vida en los pacientes adultos con PC; sin embargo, no se favorece a ningún grupo (índice de calidad de vida $\mathrm{p}=$ 0.45 y percentiles 0.48 ).

Los resultados obtenidos en nuestro estudio demuestran que, independientemente del tipo de procedimiento que se realice a este grupo en específico de pacientes, la calidad de vida después de al menos 10 años de operados es mala en comparación con la población en general sin importar el procedimiento utilizado. Lo anterior podría explicarse por la historia natural de la enfermedad, característica de esta patología.

Se necesitan estudios más controlados y con mayor seguimiento para poder definir el mejor tratamiento en este tipo de pacientes.

\section{CONCLUSIONES}

La calidad de vida de los pacientes adultos con PC GMFCS IV-V, sometidos a reconstrucción de cadera vs alargamiento de tejidos blandos más osteotomía varodesrotadora durante la infancia, es mala. No hay diferencia significativa en la calidad de vida entre ambos procedimientos a 10 años de seguimiento en los pacientes adultos.

\section{REFERENCIAS}

1. Vázquez VCC, Vidal RCA. Parálisis cerebral infantil: definición y clasificación a través de la historia. Rev Mex Ortop Ped. 2014; 16(1): 6-10.

2. Terry SC, Beaty JH. Campbells orthopaedics, infantil. Fracturas y luxaciones, parálisis cerebral. 11 edición. España: Marbán; 2015. pp. 1149-1204.

3. Robin J, Graham HK, Selber P, Dobson F, Smith K, Baker R. Proximal femoral geometry in cerebral palsy: a population-based crosssectional study. J Bone Joint Surg Br. 2008; 90(10): 1372-1379. doi: 10.1302/0301-620X.90B10.20733.

4. Noonan KJ, Walker TL, Kayes KJ, Feinberg J. Varus derotation osteotomy for the treatment of hip subluxation and dislocation in cerebral palsy: statistical analysis in 73 hips. J Pediatr Orthop B. 2001; 10(4): 279-286.

5. Moreau M, Drummond DS, Rogala E, Ashworth A, Porter T. Natural history of the dislocated hip in spastic cerebral palsy. Dev Med Child Neurol. 1979; 21(6): 749-753. doi: 10.1111/j.1469-8749.1979. tb01696.x.

6. Robb JE, Hagglund G. Hip surveillance and management of the displaced hip in cerebral palsy. J Child Orthop. 2013; 7(5): 407-413. doi: 10.1007/s11832-013-0515-6.

7. Bagg MR, Farber J, Miller F. Long-term follow-up of hip subluxation in cerebral palsy patients. J Pediatr Orthop. 1993; 13(1): 32-36. doi: 10.1097/01241398-199301000-00007.

8. Yildiz C, Demirkale I. Hip problems in cerebral palsy: screening, diagnosis and treatment. Curr Opin Pediatr. 2014; 26(1): 85-92. doi: 10.1097/MOP.0000000000000040.

9. Shore BJ, Graham HK. Management of moderate to severe hip displacement in nonambulatory children with cerebral palsy. JBJS Rev. 2017; 5(12): e4. doi: 10.2106/JBJS.RVW.17.00027.

10. Kolman SE, Ruzbarsky JJ, Spiegel DA, Baldwin KD. Salvage options in the cerebral palsy hip: a systematic review. J Pediatr Orthop. 2016; 36(6): 645-650. doi: 10.1097/ BPO.0000000000000501.

11. Maestro-Gonzalez A, Bilbao-Leon MC, Zuazua-Rico D, Fernandez-Carreira JM, Baldonedo-Cernuda RF, Mosteiro-Diaz MP. Quality of life as assessed by adults with cerebral palsy. PLoS One. 2018; 13(2): e0191960. doi: 10.1371/journal. pone.0191960.

12. Huh K, Rethlefsen SA, Wren TA, Kay RM. Surgical management of hip subluxation and dislocation in children with cerebral palsy: isolated VDRO or combined surgery? J Pediatr Orthop. 2011; 31(8): 858-863. doi: 10.1097/ BPO.0b013e31822e0261. 